\title{
Droplet desorption modes at high heat flux
}

\author{
Sergey Misyura ${ }^{1,2, *}$ and Anton Meleshkin ${ }^{1,2}$ \\ ${ }^{1}$ Institute of Thermophysics Siberian Branch, Russian Academy of Sciences, Lavrentiev Ave. 1, \\ Novosibirsk 630090, Russia \\ ${ }^{2}$ Novosibirsk State University, 2 Pirogova Street, Novosibisk 630090, Russia
}

\begin{abstract}
Nonisothermal droplet desorption of aqueous salt solution $\mathrm{H}_{2} \mathrm{O} / \mathrm{LiBr}$ during nucleate boiling was studied experimentally. A droplet was placed on a horizontal heated wall. The initial concentration of salt $C_{0}=25 \%$. The wall temperature $T_{w}=120^{\circ} \mathrm{C}$ and ambient air pressure is 1 bar. Thermal images of the temperature field on the droplet surface show an extremely non-uniform temperature field. At nucleate boiling in $\mathrm{LiBr}$ salt solution it is incorrect to predict the desorption behavior in stationary approximation. It was previously believed that the rate of evaporation does not vary with time. For the first time it is shown that the desorption rate is divided into several characteristic time intervals. These intervals is characterized by a significant change in the desorption rate.
\end{abstract}

\section{Introduction}

The droplet evaporation is observed both in nature and in technique and it is widely presented in modern literature [1-3]. Modern technologies commonly use the droplet evaporation. Evaporation of a drop and liquids were studied in [4-14]. Gas-droplet-particles flows are used in an energy sector and allow to solve environmental issues [6]. Vapor-gasdroplets flows are formed during combustion of methane hydrate. The presence of droplets leads to a significant decrease in the combustion temperature and to a decrease in the efficiency of the technology of burning natural raw materials [15-20]. The evaporation behavior depends on wall wettability [2]. The non isothermal behavior of droplet salt solution differs from one of one-component volatile liquid $[4,7,9,10]$. It is important to investigate the evaporation behavior of salt solutions for a wide range of modern technical tasks (absorption heat pumps, chemical technologies, desalination technologies). The desorption of a salt solution can be divided into several time modes. The heat transfer in salt solutions was investigated in [21,22]. The droplet evaporation depends on gas convection [23]. A formation of crystalline salt hydrates on a solution surface was considered in [24]. The velocity field measurements into gas-droplet flows using the optical methods PIV and PLIF were presented in [25]. Heat transfer and evaporation of a liquid on a structured wall is considered in [26, 27].

To date, there is very little experimental research of the droplet behavior of a salt solution during the intensive nucleate boiling. Further research is needed to develop the

* Corresponding author: $\underline{\text { misura@itp.nsc.ru }}$ 
above technologies. The purpose of this paper is to study the nucleate boiling under non isothermal desorption.

\section{Experimental data}

The experimental setup is shown in Fig. 1. Experimental studies were carried out on the horizontal surface of the heated wall (1). The working metallic section (1) was made of titanium alloy, to prevent corrosion of metal from the aggressive action of salts. A droplet (6) was placed on a horizontal heated wall. The initial concentration of salt $C_{0}=25 \%$. The wall temperature $T_{w}=125^{\circ} \mathrm{C}$ and ambient air pressure was 1 bar. The experiments were carried out at ambient air temperature $25{ }^{\circ} \mathrm{C}$, relative air humidity of $45 \%$. The initial mass concentration values $C_{0}$ of $\mathrm{LiBr}$ salt solution were determined by the densimeter. The interfacial temperature of liquid $T_{s}$ was determined by of thermal imager (NEC R500) (5). Thermocouples (4) for wall temperature measurement $T_{w}$ were located near the wall surface. The temperature difference on the wall surface did not exceed $1-2{ }^{\circ} \mathrm{C}$. A change in the solution mass was recorded by means of the balance (2). The initial droplet volume was $V_{0}=100 \mu \mathrm{l}$.

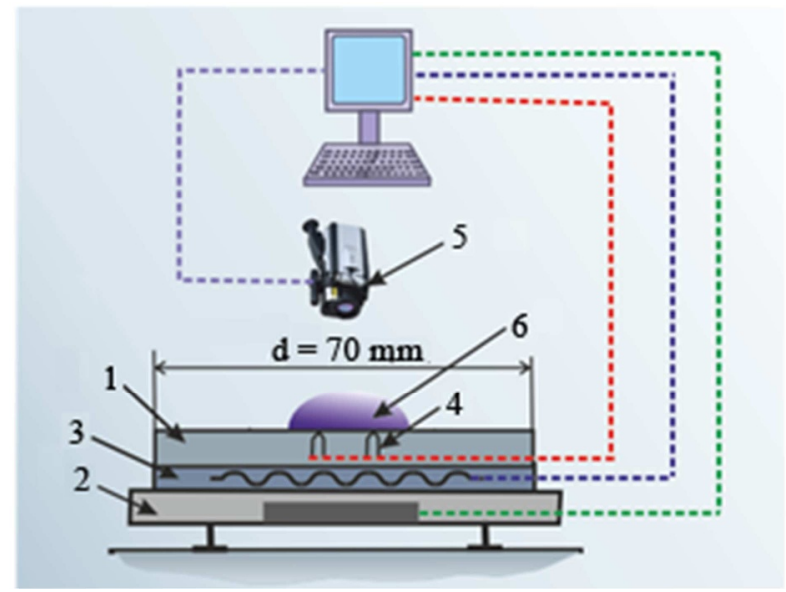

Fig. 1. The scheme of the experimental setup: 1 - the working section (titanium cylinder); 2 - scales; 3 - heater; 4 - thermocouples; 5 - the thermal imager; 6 - a droplet of $\mathrm{LiBr}$ salt solution.

The droplet evaporation rate $j$ was determined as $j=\Delta m / \Delta t$ ( $m$ is the liquid mass and $t$ is the time). The maximum error of measurement of $j$ was $17-20 \%$ for a high salt concentration before crystallization onset and it is related to the measurement error of $\Delta m$. Mass concentration of salt in the salt solution was determined as $C_{1 \mathrm{i}}=m_{\text {salt }} / m$, ( $m$ is the mass of aqueous salt solution, $m_{\text {salt }}$ is the mass of salt in the solution).

The thermal image of nucleate boiling of a droplet of $\mathrm{LiBr}$ salt solution $\left(T_{w}=120{ }^{\circ} \mathrm{C}\right)$ is shown in Fig. 2 (a) for $t=10 \mathrm{~s}$. Arrow 1 indicates a dry spot at the bottom of a "burst" bubble (the spot is shown black). Arrow 2 demonstrates a thin desorption layer near the bubble bottom; Arrow 3 shows a cooler liquid that filled a dry spot. After the bubble collapse the solution covers the dry spot and reduces wall temperature. Aqueous salt solution without vapor bubbles is shown by the arrow 4. As can be seen from Fig. 2, the temperature field on the drop surface is strongly uneven due to the growth and the destruction of bubbles and convection into the droplet. The thermal images of growth of crystalline salt hydrates are shown in Fig. 2 (b). We can clearly observe formation of the "rod" crystalline structures for $t=50 \mathrm{~s}$. 


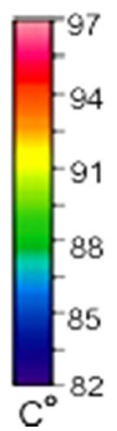

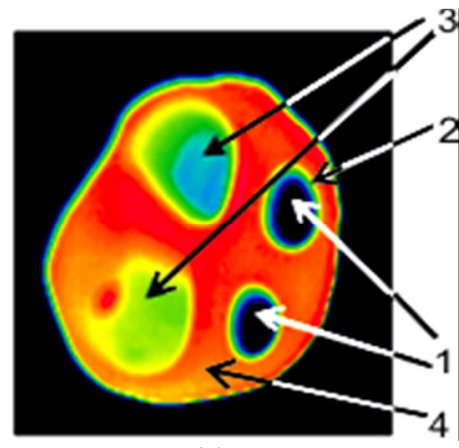

(a)

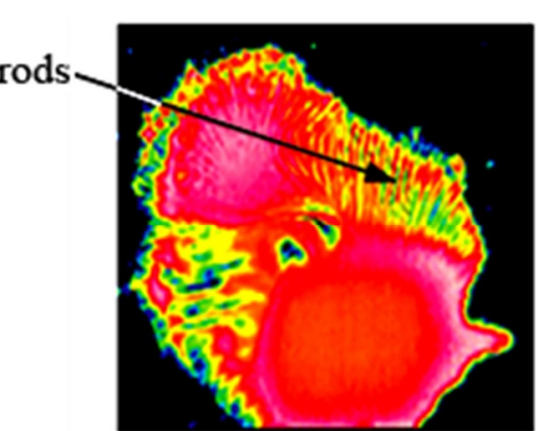

(b)

Fig. 2. a) Thermal image at nucleate boiling of a droplet of $\mathrm{LiBr}$ salt solution; b) Thermal image of crystallization process $\left(C_{0}=25 \%, T_{w}=125^{\circ} \mathrm{C}, V_{0}=100 \mu \mathrm{l}\right)$.

The behavior of the evaporation rate for the droplet of aqueous salt solution is shown in Fig. 3. There are 5 characteristic time intervals for $\mathrm{LiBr}$ droplet: 1) The initial heating and expansion of the drop on the hot wall (the first 5-7 s) (first mode). As a result of the nucleate boiling, the droplet radius increases twice, and the droplet area increases significantly. 2) Further, the droplet diameter is constant during the whole evaporation time. The growth of the droplet area and the heating of the solution lead to a multiple increase in the evaporation rate. 3) The wall fails to compensate cooling due to thermal inertia of the metal wall and because of a sharp increase in $j$. As a result, the temperature under the $\mathrm{LiBr}$ solution drops and $j$ decreases. 4) The wall temperature under the droplet increases after a few seconds, and $j$ also increases for the mode. 5) A further increase in salt concentration leads to a significant decrease in the evaporation rate for mode 5.

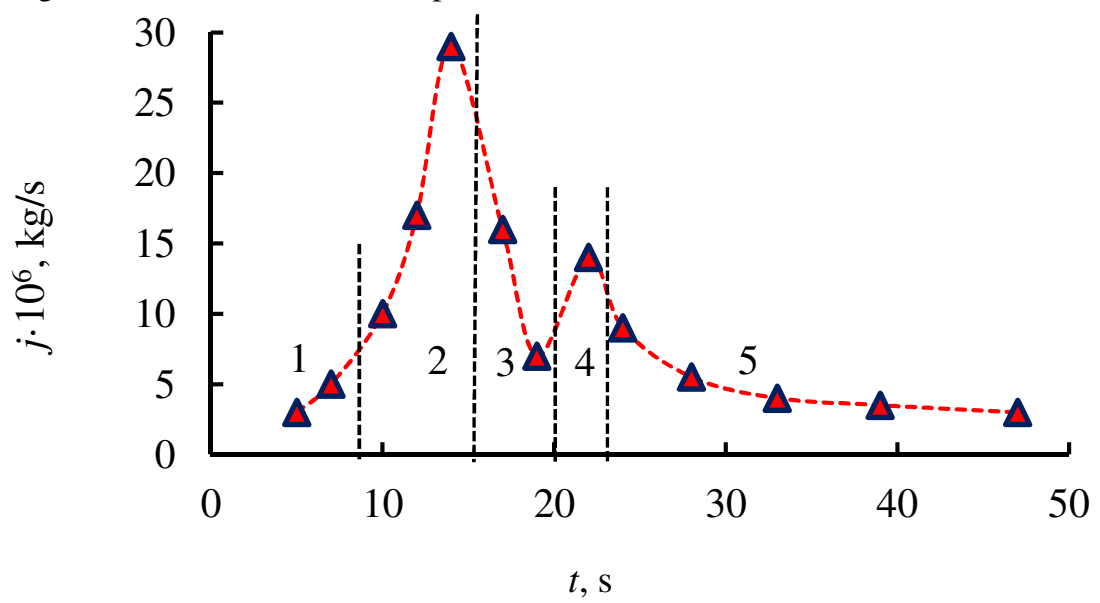

Fig. 3. Change in the evaporation rate $j$ of $\mathrm{LiBr}$ salt solution with time $\left(C_{0}=25 \%, T_{w}=125^{\circ} \mathrm{C}\right.$, $\left.V_{0}=100 \mu \mathrm{l}\right)$.

Usually the calculation of desorption rate is realized under the assumption of constancy $j$ with time. The novelty of this paper is that the unacceptability of this assumption is shown. Desorption rate is divided into 4 characteristic time regimes with increasing time and salt concentration. For each indicated regime, there are different boundary conditions for the wall temperature and for the desorption rate. The results of these experimental studies are important for a wide range of technical problems (desorption in heat pumps, chemical technologies, desalination technologies) and the obtained experimental data can be used for the correct modeling nonisothermal desorption. 


\section{Conclusions}

Nonisothermal droplet desorption of aqueous salt solution of $\mathrm{H}_{2} \mathrm{O} / \mathrm{LiBr}$ during intensive nucleate boiling was studied experimentally.

The desorption rate of a droplet of $\mathrm{LiBr}$ salt solution is divided into 5 characteristic time regimes with increasing salt concentration. For each mode, there are different boundary conditions for the wall temperature $T_{w}$ under the droplet and for desorption rate $j$.

The temperature field on the drop surface is strongly uneven due to the growth and the destruction of bubbles and convection into the sessile droplet. Dendritic crystalline hydrates of the salt grow on the surface of the drop.

The results of these experimental studies are important for a wide range of technical tasks: the desorption of aqueous salt solutions in adsorption heat pumps, chemical technologies, desalination technologies.

Experiments related to measurements of evaporation rate at nucleate boiling were carried out at the Kutateladze Institute of Thermophysics SB RAS and financially supported by the Russian Science Foundation (project number 15-19-10025). Experiments related to crystallization and computer calculations were carried out in Novosibirsk State University.

\section{References}

1. G.V. Kuznetsov et. al., Thermophysics and Aeromechanics 23, 17 (2016)

2. G.V. Kuznetsov et. al., J. of Engineering Thermophysics 89, 317 (2016)

3. S.Y. Misyura, Exp. Therm. Fluid Sci. 84, 190 (2017)

4. V.E. Nakoryakov et al., Doklady Physics 59, 441 (2014)

5. G.V. Kuznetsov et. al., Int. J. Heat Mass Transfer 96, 20 (2016)

6. P.A. Strizhak et. al., J. of Hazardous Materials 338, 148 (2017)

7. S.Y. Misyura, Scientific Reports 7, 14759 (2017)

8. V.V. Kuznetsov et al., J. Eng. Thermophys. 24, 357 (2015)

9. S.Y Misyura, Int. J. of Heat and Mass Transfer 125, 610 (2018)

10. S.Y Misyura, Int. J. of Heat and Mass Transfer 116, 667 (2018)

11. O.A. Kabov et. al., Nanoscale and Microscale Thermophysical Engineering 21, 60 (2017).

12. S.Y Misyura, Int. J. of Thermal Sciences 124, 76 (2018)

13. S.Semenov, Adv. Colloid Interface Sci. 206, 382 (2014)

14. S.Y. Misyura, Chem. Eng. Research and Design 126, 153 (2017)

15. S.Y. Misyura, I.G. Donskoy, Chem. Eng. Sci. 148, 65 (2016)

16. S.Y. Misyura, V.E. Nakoryakov, Energy and Fuels 27, 7089 (2013)

17. M.E. Semenov et. all., Journal of Thermal Analysis and Calorimetry 119, 757 (2015)

18. S.Y. Misyura, Energy 103, 430 (2016)

19. V.E. Nakoryakov et. al., J. of Engineering Thermophysics 22(3), 169 (2013)

20. V.E. Nakoryakov et. al., J. of Engineering Thermophysics 22(2), 87 (2013)

21. S.Y. Misyura, Applied Thermal Engineering 113, 472 (2017)

22. S.Y. Misyura, Appl. Therm. Eng. 139, 203 (2018)

23. F. Carle et al., Intern. J. of Therm. Sci. 101, 35 (2016) 
24. S.Y. Misyura, Crystal Growth and Design 18, 1327 (2018)

25. G.V. Kuznetsov et. al., Appl. Therm. Eng. 131, 340 (2018)

26. S.Y. Misyura, Int. Comm. Heat Mass Transfer 96, 7 (2018)

27. S.Y. Misyura, Nanoscale and Microscale Thermophysical Engineering 22(3), 213 (2018). 